\title{
Posterior to the ridge laser treatment for severe stage 3 retinopathy of prematurity
}

AL Ells',2, GA Gole ${ }^{3}$, P Lloyd Hildebrand ${ }^{4}$, A Ingram ${ }^{1}$, CM Wilson ${ }^{5,6}$ and R Geoff Williams ${ }^{2,7}$

\begin{abstract}
Background Current methods of treating the avascular retina with laser photocoagulation for severe retinopathy of prematurity (ROP) are not completely effective in the reduction of visual morbidity. We report a case series in which additional laser treatment, called 'posterior laser', was delivered posterior to the neovascular ridge, for eyes with severe stage 3 ROP in zone II with avascular retina posterior to the ridge.

Design Retrospective chart review.

Participants Infants who underwent laser treatment, posterior to the neovascular ridge for severe ROP at the Alberta Children's Hospital, between January 2005 and October 2008.
\end{abstract}

Methods Charts were reviewed for 18 eyes of 11 patients and collected information included demographic data, clinical examination results, and digital retinal images.

Main Outcome Measures Structural and functional outcomes of treatment. Results Four (22\%) of 18 eyes received 'posterior laser' as primary treatment and the remainder of eyes $(\mathbf{7 8 \%})$ received 'posterior laser' following previous laser photocoagulation anterior to the neovascular ridge. Mean birthweight was $688 \mathrm{~g}$ (552-930) and mean gestational age was 24 weeks (2328). There were no complications because of the posterior laser treatment. In all, 16 of 18 eyes experienced rapid regression of the ridge and subsequent decrease in vascular dilation and tortuosity within 1 week. Two eyes required vitrectomy for $4 \mathrm{~A}$ retinal detachment; however, no eyes developed stage 4B ROP.

Conclusion Posterior to the ridge laser in the setting of the morphological criteria described had no increased safety concerns and resulted in rapid regression of ROP with good outcomes.

Eye (2013) 27, 525-530; doi:10.1038/eye.2012.302; published online 18 January 2013

Keywords: laser photocoagulation; neovascular ridge; posterior laser treatment; retinopathy of prematurity

\section{Introduction}

Despite $>65$ years of clinical and basic science research, retinopathy of prematurity (ROP) continues to be the world's leading cause of preventable blindness in children. ${ }^{1}$ With improvements in prenatal care and availability of neonatal intensive care units, ROP still presents challenges in highly developed countries and is growing rapidly in middle development countries. ${ }^{1}$ The pathogenesis of $\mathrm{ROP}$ is attributed to developing premature retinal vessels responding abnormally to both hyperoxia and hypoxia, resulting in pathological vasoproliferative lesions. Morphologic characteristics of this progressive retinopathy are used to stage the severity of disease. When enough high-risk morphological features of severe, acute, vascularly active disease are present, infants benefit from laser photocoagulation to the premature avascular retina. ${ }^{2}$ In addition to the growing prevalence of ROP, there has been an increase in the number of cases of severe stage 3 ROP in which traditional laser treatment does not reliably halt the progression of disease. ${ }^{1,3}$ While timely laser treatment is efficacious in preventing permanent blindness, ${ }^{2,4}$ current standard methods of treating the avascular retina are not completely effective in reducing visual morbidity in this high-risk group. In data published from the early treatment of ROP
${ }^{1}$ Ells Retina Centre, Calgary, Alberta, Canada

${ }^{2}$ Faculty of Medicine, Department of Ophthalmology, University of Calgary, Calgary, Alberta, Canada

${ }^{3}$ Discipline of Paediatrics and Child Health, University of Queensland, Royal Children's Hospital, Brisbane, Queensland, Australia

${ }^{4}$ Department of Ophthalmology, University of Oklahoma, Oklahoma City, OK, USA

${ }^{5}$ Department of Optometry and Visual Science, City University, London, UK

${ }^{6}$ Department of Ophthalmology, St Mary's Hospital, London, UK

${ }^{7}$ Calgary Retina Consultants, Calgary, Alberta, Canada

Correspondence: AL Ells, Ophthalmology, Ells Retina Centre, Highstreet House, 933-17th Avenue SW, Suite 344, Calgary, Alberta T2T 5R6, Canada Tel: + 1403245 3171; Fax: + 14032454205 E-mail: annaells@mac.com

Received: 20 July 2012 Accepted in revised form: 15 December 2012 Published online: 18 January 2013 
(ETROP) clinical trial, 63 (15.7\%) of 401 high-risk pre-threshold patients randomized for early standard laser treatment of the avascular retina progressed to fibrovascular changes and stage 4 or stage 5 retinal detachment. ${ }^{3}$ In all, 75 eyes of 57 patients required vitreoretinal surgery, only 13 (17\%) eyes had favorable visual outcome at $41 / 2$ years. We report a case series of a modified laser treatment protocol for eyes with severe zone II, stage 3 ROP. Laser photocoagulation was delivered posterior to the demarcation between vascularized and non-vascular retina ('posterior laser') in addition to usual ablation of avascular retina anterior to the ridge. Posterior laser targeted retina immediately posterior to the ridge, may eliminate residual areas of ischemic retina ${ }^{5-7}$ and may facilitate regression of ROP. We report the anatomical outcomes, early visual outcomes, and ocular safety of a cohort of 18 eyes treated using posterior laser for severe stage 3 ROP.

\section{Materials and methods}

This retrospective case series reviewed all babies treated with retinal laser between January 2005 and October 2008 at the Alberta Children's Hospital, Calgary, Alberta, Canada. Demographic data and examination findings for each infant were retrospectively reviewed using the Telemedicine for ROP in Calgary (TROPIC) database. Demographic information included clinical binocular indirect ophthalmoscopic examination results and standardized wide-field RetCam (Clarity Medical Systems, Pleasanton, CA, USA) digital retinal images documenting before and after laser retinopathy. Alberta Children's Hospital research review board granted approval for the database and the study adhered to the tenets of the Declarations of Helsinki.

Morphologic criteria identified for posterior laser treatment included infants with severe zone II, stage 3 ROP consisting of: (1) a thick, neovascular ridge; (2) $>4$ confluent temporal clock hours of neovascular ridge; (3) plus disease in two temporal quadrants (Figure 1a). Furthermore, to qualify for this treatment, a minimum distance of $3000 \mu \mathrm{m}$ (two disc diameters) between the fovea and temporal ridge was required (Figure 1b). Minimal temporal retinal traction was allowed. Text boxes 1 and 2 summarize the morphological criteria. These treatment criteria were required for infants receiving primary treatment and infants who had previously undergone standard, avascular retinal laser treatment. In the previously treated group, ROP had to have progressed for at least 2 weeks following initial laser, despite adequate laser to the avascular retina during the initial treatment.

Posterior laser treatment was performed within $48 \mathrm{~h}$ of the examination in which the patient met treatment criteria, as outlined in Table 1. The treatment was fully described to parents and they were informed that this was not the standard of care. Treatment was performed using an indirect infrared, diode laser $(810 \mathrm{~nm})$ through a 25 diopter condensing lens for visualization, with initial power settings of $200 \mathrm{~mW}$, duration of $200 \mathrm{~ms}$. Laser power was increased up to $450 \mathrm{~mW}$ until there was evidence of light retinal blanching. Two or three rows (based on the available distance from ridge to center of fovea) of laser burns were delivered to the posterior aspect of the ridge in the area of vascularized retina, avoiding blood vessels (Figures 2a and b). Each eye was examined on the day of diagnosis, before treatment, 3-5 days post treatment and then weekly (Figure 2c), until ROP regressed. Follow-up examinations were then performed at 6 weeks then 3-month intervals for the first year following treatment.

\section{Results}

A total of 18 eyes of 11 babies were treated using the posterior laser method. The remaining 4 eyes of these 11 infants were treated with laser to the avascular, anterior

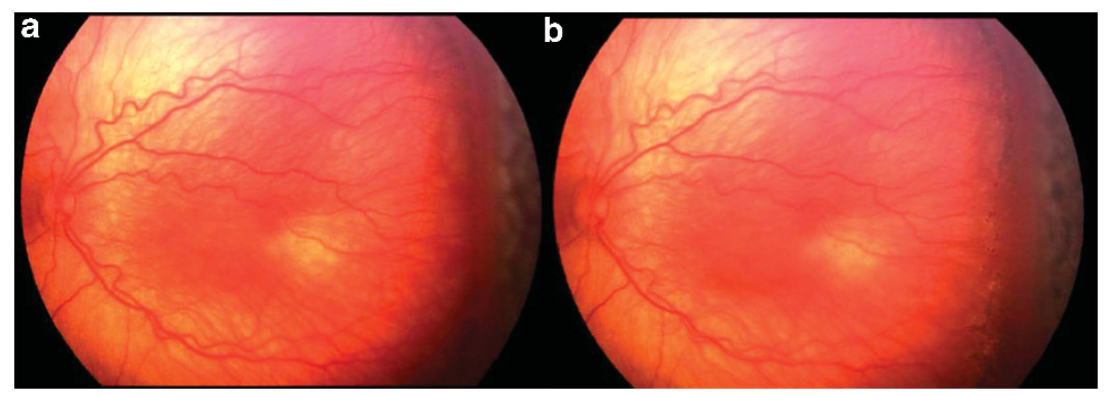

Figure 1 (a) Note the temporal, confluent, elevated, thick neovascular ridge; plus disease in temporal two quadrants. Near-confluent laser pattern has been performed to avascular retina. (b) A wide-field digital image taken 1 week after laser treatment. Note light laser scars posterior to neovascular ridge. There is a minimum distance of $3000 \mu \mathrm{m}$ (two disc diameters) between the fovea and the temporal ridge. 
Table 1 Morphological criteria for patients treated with laser posterior to the neovascular ridge for severe ROP in this series

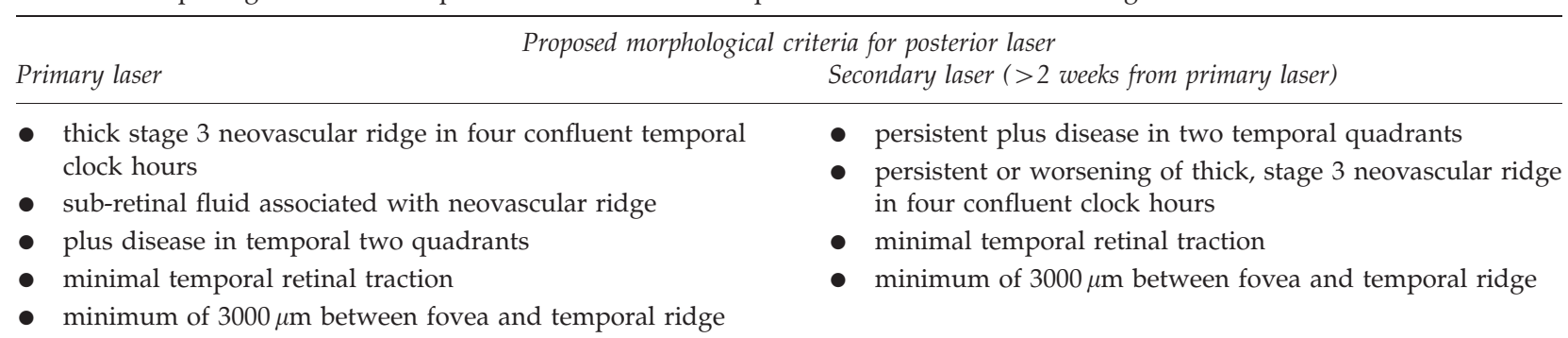

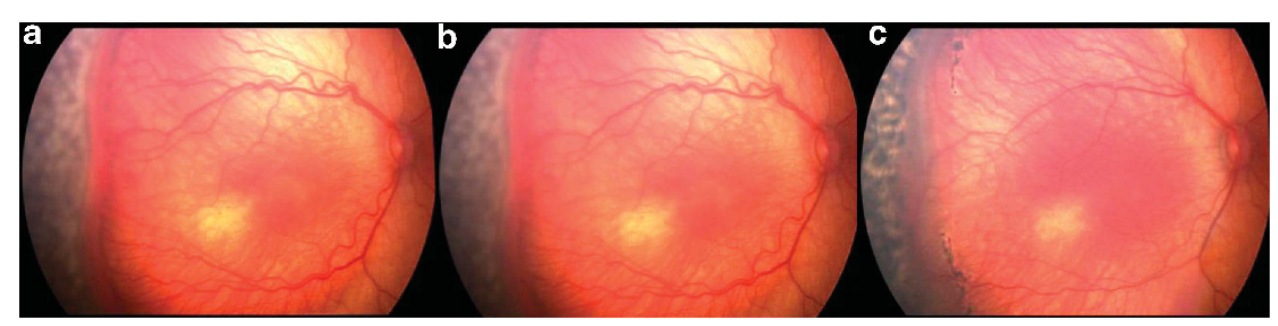

Figure 2 (a) Demonstrates primary laser applied to the avascular retina, anterior to the neovascular ridge. (b) Digital image taken immediately after laser has been delivered to retina immediately posterior to the neovascular ridge. Note the light burns. (c) Two weeks after posterior laser. Note progressive thinning of neovascular membrane, decrease in arborization of vessels just posterior to the ridge, and decrease in plus disease in temporal two quadrants.

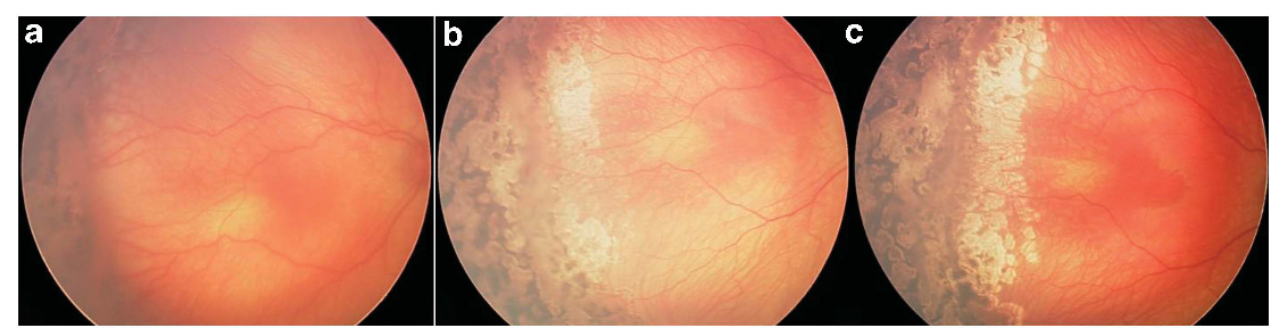

Figure 3 (a) A wide-field digital image 14 days after primary laser to the avascular retina. Note thick, confluent, persistent neovascular ridge. Laser was applied immediately posterior to the ridge and to areas of non-lasered retina anterior to the ridge. (b) Image was taken 3 weeks later. Note laser scars of both anterior and posterior laser. Note temporal thin, atrophic membrane. (c) Image was taken 3 weeks later. Note more complete regression of membrane.

retina only. Nine infants were Caucasian, one Asian, and one Native American. Four eyes had laser photocoagulation posterior to the ridge as primary treatment and 14 eyes had posterior laser following previous laser photocoagulation anterior to the ridge. Mean birth weight was $688 \mathrm{~g}$ (552-930 g), and mean gestational age at birth was 24 weeks (23-28 weeks). Mean follow-up was 24 months (14-40 months). Mean chronological age at time of initial posterior laser was 80 days (64-105 days) and secondary posterior laser treatment was 100 days (83-129 days). Mean post menstrual age at the time of posterior laser was 37 weeks (34-41 weeks). The mean post menstrual age of treatment when divided by primary and secondary treatment group was 36 and 38 weeks, respectively. Best-corrected visual acuities (BCVA) were able to be measured, using
Teller Acuity Cards for 13 eyes at the final follow-up visit. Mean BCVA was 20/84 (20/60 to 20/360). Despite posterior laser, two eyes had ROP that continued to progress, requiring vitrectomy for $4 \mathrm{~A}$ detachment, both were in the secondary treatment group. One of these eyes has BCVA of 20/60 after 23 months of follow-up (Figure 3a) and the other infant was preverbal and vision was central, steady and maintained. No infants developed stage 4B ROP. The regression of the heavy neovascular ridge was rapid in the remaining 16 eyes with evidence of decreased vascular dilation, tortuosity, thickness, and redness of neovascular ridge within a week of treatment. These eyes developed thinning, atrophic membranes in place of the neovascular ridge that could be seen between the anterior and posterior placement of laser burns 3 to 4 weeks after treatment 
(Figures $3 \mathrm{~b}$ and c). During this study period, 102 eyes of 52 infants were treated for severe ROP and 18 (17.6\%) of these eyes received posterior laser treatment and only 2 eyes progressed to stage $4 \mathrm{~A}$ detachment.

During the 46 months before the implementation of posterior laser treatment, 116 eyes of 58 infants were treated for severe ROP and 7 of these eyes went on to stage 4 retinal detachments (5 eyes developed $4 \mathrm{~A}$ and 2 eyes developed $4 \mathrm{~B}$ retinal detachments).

None of the study patients suffered complications of posterior laser including preretinal, intraretinal, subretinal, vitreous hemorrhages, or hyphema. No patients developed cataracts, macular holes, or scarring into the parafoveal area.

\section{Discussion}

ROP continues to be a leading cause of preventable childhood blindness and vision loss worldwide, despite evidence-based recommendations for screening examinations and timely laser treatment. The understanding of ROP has advanced significantly in the last several decades yet the approach to laser treatment has remained relatively unchanged since its widespread use began in the early 1990s. Can we do better with laser treatment to prevent visual loss from ROP using available evidence from epidemiologic studies, clinical observation, and the laboratory?

The cryotherapy for ROP study (CRYO-ROP) and ETROP studies have demonstrated that visual outcome may be poor for zone II ROP. ${ }^{2,8}$ Twenty percent of stage 3, zone II eyes treated in the CRYO-ROP study went on to unfavorable outcome, or vision $<20 / 200 .{ }^{9}$ In the ETROP study, $15.3 \%$ of zone II, stage 3 eyes with plus disease had an unfavorable acuity at 9 months of age. ${ }^{4}$ ETROP defined an unfavorable visual acuity as $<1.85 \mathrm{cyc} / \mathrm{deg}$ or vision $<20 / 320$ Snellen equivalent. Treatable zone II, stage 3 ROP may have a spectrum of severity at the time of treatment and may have corresponding favorable and unfavorable visual outcomes. We believed that modification of traditional laser technique may be warranted to improve outcomes in the most severe presentation of zone II, stage 3 ROP.

In very severe zone II, stage $3 \mathrm{ROP}$, the standard nearconfluent laser to avascular retina does not consistently induce rapid regression of the thick, dilated, neovascular ridge, and prevent cicatricial structural changes,

resulting in vision loss, as reported by ETROP. In the past 5 years, we have observed that standard laser treatment may result in minimal regression of the thick, confluent, elevated neovascular ridge in the temporal quadrants and these eyes often progress to develop cicatricial changes resulting in collapse and straightening of the temporal arcade angle and vessels. Frequently, the thick neovascular ridge continued to extend into the vitreous, evolving into a stage 4 retinal detachment requiring vitreoretinal surgery. These observations prompted us to develop a set of clinical morphological features for which the treating surgeon should consider posterior laser treatment. It is important to emphasize that the ROP addressed in this approach is not aggressive posterior ROP, but rather a severe form of the zone II, stage 3 ROP that requires laser treatment. ${ }^{10}$

We hypothesize that the mechanism of action of posterior laser works in several ways: (1) laser destroys the retinal pigment epithelial barrier acutely and until the barrier heals, subtle non-proteinaceous subretinal fluid will be drawn out rapidly by the oncotic pressure of the choroid, thus absorbing the subretinal fluid at the retinal interface associated with the neovascular ridge; ${ }^{11,12}$ (2) the laser burn acts as a mechanical barrier to detachment by creating chorioretinal adhesions; ${ }^{13-15}$ (3) photocoagulation burns may destroy the vasogenic spindle cells, astrocytes, and other pluripotential cells near the posterior aspect of the neovascular ridge thereby reducing vascular endothelial growth factor (VEGF) production; $5,6,16-19$ and (4) photocoagulation treats those discrete areas of capillary closure that occur posterior to the ridge (as seen on fluorescein angiography) that may act as the source for ongoing VEGF production in eyes, which have not responded to laser ablation of the peripheral retina anterior to the ridge.

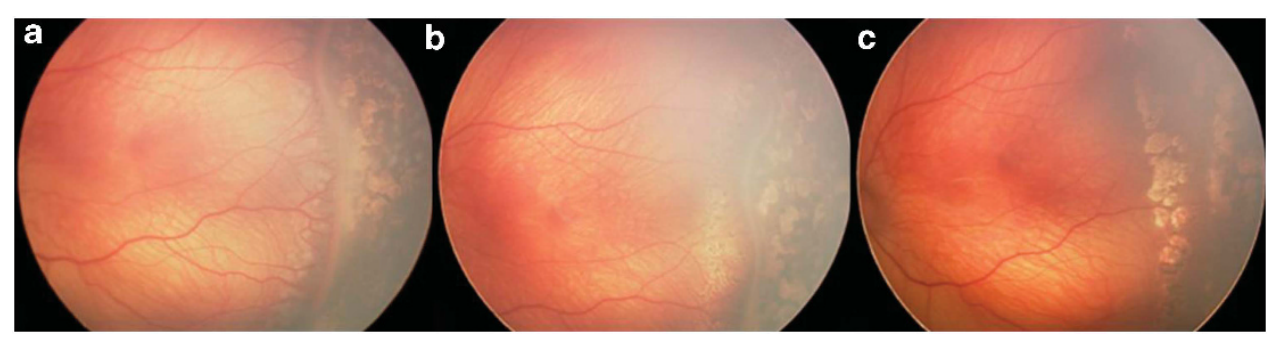

Figure 4 (a) Shows posterior light laser burns immediately after procedure. This secondary laser was performed 24 days after initial laser procedure to avascular retina. (b) Image was taken 2 weeks after posterior laser. Note thin, atrophic membrane and decrease in posterior arborized vessels. (c) Shows the membrane of almost completely regressed with no cicatricial changes present. 
Treatment of the vascularized premature retina was first reported in 1978 by Hindle and Leyton ${ }^{20}$ using cryotherapy to the neovascular ridge and adjacent areas both anterior and posterior to the ridge. Several other reports of laser therapy to the posterior aspect of the ridge for posterior location of zone II, stage 3 ROP have also been reported. ${ }^{21-23}$ Results of this retrospective review of 18 eyes treated with posterior laser shows that the treatment was safe and resulted in good anatomical and visual outcomes (Figures $4 \mathrm{a}-\mathrm{c}$ ). The anatomical success is similar to previously published reports of laser to the vascular retina. ${ }^{22-25}$ Our case series proposes criteria to select eyes that we believe would benefit from this alternative technique of laser treatment. We found a decrease in the overall progression to stage 4 retinal detachments when compared with the equivalent time period before delivering posterior laser technique.

We recognize that the study findings are limited by its observational, retrospective design. This case series report was not designed to test whether posterior laser was more efficacious than standard laser treatment for ROP and it was not specifically designed to test the validity of the proposed morphological criteria. It is difficult to know the extent of structural and visual benefit of posterior laser without a standard of care control arm and strong statistical support. Other limitations include the relatively small cohort and the inherent difficulty of quantifying the characteristics of severe zone II, stage 3 disease or 'burden of disease' that may benefit from posterior laser. A subset of infants in this review received laser posterior to the ridge as secondary treatment after progression following primary laser ablation of peripheral avascular retina. Skip areas or area's of avascular retina without laser, were also lasered at the time of laser posterior to the ridge. This could contribute to regression of ROP following the secondary laser procedure.

Laser photocoagulation posterior to the ridge is not usually performed because of concerns for inducing complications and damaging viable retina as well, a limited evidence base is not generally advocated in guideline statements. Laser close to the posterior aspect of the neovascular ridge may result in retinal or vitreous hemorrhage and if there is a sub-clinical stage $4 \mathrm{~A}$ retinal detachment present, laser may cause a retinal break. We had no complications associated with this procedure and great care was taken in delivering very light posterior laser burns and excluding those eyes with any signs of cicatricial ROP to suggest impending $4 \mathrm{~A}$ retinal detachment.

Anti-VEGF therapies have not shown to be more efficacious than laser photocoagulation in zone II ROP and intra-vitreal safety profiles in premature infants have also not been clearly studied. ${ }^{26,27}$
Our case series does not include any infants treated with anti-VEGF drugs.

In this study, we describe morphological criteria for consideration of posterior laser as a primary or secondary laser procedure in select cases of severe zone II, stage 3 ROP. Laser photocoagulation to the retina immediately posterior to the ridge in the presence of these criteria may halt progression of the disease safely, quickly, and effectively and may minimize visual loss from cicatricial macular changes or avoid progression to stage 4 ROP warranting vitreoretinal surgery.

\section{Summary}

\section{What was known before}

- In addition to the growing prevalence of ROP, there has been an increase in the number of cases of severe stage 3 ROP in which traditional laser treatment does not reliably halt the progression of disease.

- While timely laser treatment is efficacious in preventing permanent blindness, current standard methods of treating the avascular retina are not completely effective in reducing visual morbidity in this high-risk group.

What this study adds

- We report a case series in which additional laser treatment, called 'posterior laser', was delivered posterior to the neovascular ridge, for eyes with severe stage 3 ROP in zone II with avascular retina posterior to the ridge.

\section{Conflict of interest}

The authors declare no conflict of interest.

\section{References}

1 Gilbert C, Fielder A, Gordillo L, Quinn G, Semiglia R, Visintin $\mathrm{P}$ et al. Characteristics of infants with severe retinopathy of prematurity in countries with low, moderate, and high levels of development: implications for screening programs. Pediatrics 2005; 115(5): e518-e525.

2 Cryotherapy for Retinopathy of Prematurity Cooperative Group. Multicenter trial of cryotherapy for retinopathy of prematurity: ophthalmological outcomes at 10 years. Arch Ophthalmol 2001; 119(8): 1110-1118.

3 Repka MX, Tung B, Good WV, Shapiro M, Capone Jr, A, Baker JD et al. Outcome of eyes developing retinal detachment during the early treatment for retinopathy of prematurity study (ETROP). Arch Ophthalmol 2006; 124(1): 24-30.

4 Good WV. Early Treatment for Retinopathy of Prematurity Cooperative Group. Final results of the early treatment for retinopathy of prematurity (ETROP) randomized trial. Trans Am Ophthalmol Soc 2004; 102: 233-48; discussion 248-50.

5 Smith LE. IGF-1 and retinopathy of prematurity in the preterm infant. Biol Neonate 2005; 88(3): 237-244.

6 Smith LE. Pathogenesis of retinopathy of prematurity. Growth Horm IGF Res 2004; 14(Suppl A): S140-S144. 
7 Kushner BJ, Essner D, Cohen IJ, Flynn JT. Retrolental fibroplasia. II. pathologic correlation. Arch Ophthalmol 1977; 95(1): 29-38.

8 Palmer EA, Hardy RJ, Dobson V, Phelps DL, Quinn GE, Summers CG et al. 15-Year outcomes following threshold retinopathy of prematurity: final results from the multicenter trial of cryotherapy for retinopathy of prematurity. Arch Ophthalmol 2005; 123(3): 311-318

9 Cryotherapy for Retinopathy of Prematurity Cooperative Group. Multicenter trial of cryotherapy for retinopathy of prematurity: natural history ROP: ocular outcome at 5(1/2) years in premature infants with birth weights less than 1251g. Arch Ophthalmol 2002; 120(5): 595-599.

10 International Committee for the Classification of Retinopathy of Prematurity. The international classification of retinopathy of prematurity revisited. Arch Ophthalmol 2005; 123(7): 991-999.

11 Lanzetta P, Furlan F, Morgante L, Veritti D, Bandello F. Nonvisible subthreshold micropulse diode laser $(810 \mathrm{~nm})$ treatment of central serous chorioretinopathy. A pilot study. Eur J Ophthalmol 2008; 18(6): 934-940.

12 Frambach DA, Marmor MF. The rate and route of fluid resorption from the subretinal space of the rabbit. Invest Ophthalmol Vis Sci 1982; 22(3): 292-302.

13 Schmidt D. Macular-threatening traction detachment of the retina in diabetic proliferative retinopathy, treated by laser. Int Ophthalmol 1997; 21(2): 99-106.

14 Kain HL. Chorioretinal adhesion after argon laser photocoagulation. Arch Ophthalmol 1984; 102(4): 612-615.

15 Menchini U, Trabucchi G, Brancato R, Cappellini A. Can the diode laser (810nm) effectively produce chorioretinal adhesion? Retina 1992; 12(3 Suppl): S80-S86

16 Chan-Ling T, Chu Y, Baxter L, Weible Ii M, Hughes S. In vivo characterization of astrocyte precursor cells (APCs) and astrocytes in developing rat retinae: differentiation, proliferation, and apoptosis. Glia 2009; 57(1): 39-53.

17 Chan-Ling T, Page MP, Gardiner T, Rosinova E, Hughes S. Desmin ensheathment ratio as an indicator of vessel stability: evidence in normal development and in retinopathy of prematurity. Am I Pathol 2004; 165(4): 1301-1313.

18 Pierce EA, Foley ED, Smith LE. Regulation of vascular endothelial growth factor by oxygen in a model of retinopathy of prematurity. Arch Ophthalmol 1996; 114(10): 1219-1228.

19 Pierce EA, Avery RL, Foley ED, Aiello LP, Smith LE. Vascular endothelial growth factor/vascular permeability factor expression in a mouse model of retinal neovascularization. Proc Natl Acad Sci USA 1995; 92(3): 905-909.

20 Hindle NW, Leyton J. Prevention of cicatricial retrolental fibroplasia by cryotherapy. Can J Ophthalmol 1978; 13(4): 277-282.

21 Axer-Siegel R, Maharshak I, Snir M, Friling R, Ehrlich R, Sherf I et al. Diode laser treatment of retinopathy of prematurity: anatomical and refractive outcomes. Retina 2008; 28(6): 839-846.

22 Axer-Siegel R, Snir M, Cotlear D, Maayan A, Frilling R, Rosenbaltt I et al. Diode laser treatment of posterior retinopathy of prematurity. Br J Ophthalmol 2000; 84(12): 1383-1386.

23 O'Keefe M, Burke J, Algawi K, Goggin M. Diode laser photocoagulation to the vascular retina for progressively advancing retinopathy of prematurity. Br J Ophthalmol 1995; 79(11): 1012-1014.

24 Steinmetz RL, Brooks Jr., HL. Diode laser photocoagulation to the ridge and avascular retina in threshold retinopathy of prematurity. Retina 2002; 22(1): 48-52.

25 Axer-Siegel R, Snir M. Diode laser photocoagulation to the ridge and avascular retina in threshold retinopathy of prematurity. Retina 2003; 23(1): 126-127; author reply 127.

26 Darlow BA, Ells AL, Gilbert CE, Gole GA, Quinn GE. Are we there yet? Bevacizumab therapy for retinopathy of prematurity. Arch Dis Child Fetal Neonatal Ed 2011; e-pub ahead of print 30 December 2011; doi:10.1136/archdischild2011-301148.

27 Gole GA, Camuglia JE, Ells AL. Bevacizumab for retinopathy of prematurity. N Engl J Med 2011; 364(24): 2360-2361. 